\title{
$\beta$-Adrenergic Receptors : New Target in Breast Cancer
}

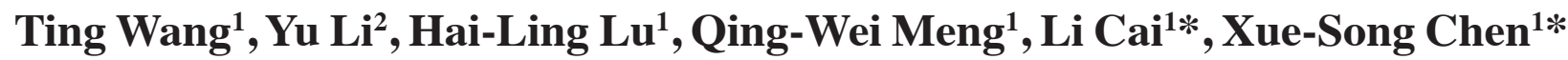

\begin{abstract}
Background: Preclinical studies have demonstrated that $\beta$-adrenergic receptor antagonists could improve the prognosis of breast cancer. However, the conclusions of clinical and pharmacoepidemiological studies have been inconsistent. This review was conducted to re-assess the relationship between beta-adrenoceptor blockers and breast cancer prognosis. Materials and Methods: The literature was searched from PubMed, EMBASE and Web of Nature (Thompson Reuters) databases through using key terms, such as breast cancer and betaadrenoceptor blockers. $\underline{\text { Results: }}$ Ten publications met the inclusion criteria. Six suggested that receiving betaadrenoceptor blockers reduced the risk of breast cancer-specific mortality, and three of them had statistical significance (hazard ratio $(\mathrm{HR})=0.42 ; 95 \% \mathrm{CI}=0.18-0.97 ; p=0.042)$. Two studies reported that risk of recurrence and distant metastasis (DM) were both significantly reduced. One study demonstrated that the risk of relapsefree survival (RFS) was raised significantly with beta-blockers $(\mathrm{BBS})(\mathrm{HR}=0.30 ; 95 \% \mathrm{CI}=\mathbf{0 . 1 0 - 0 . 8 7} ; \boldsymbol{p}=\mathbf{0 . 0 2 7})$. One reported longer disease-free interval $(\log \operatorname{Rank}(\mathrm{LR})=6.658 ; p=0.011)$ in $\mathrm{BBS}$ users, but there was no significant association between overall survival $(\mathrm{OS})$ and $B B S(H R=0.35 ; 95 \% C I=0.12-1.0 ; p=0.05)$ in five studies. Conclusions: Through careful consideration, it is suggested that beta-adrenoceptor blockers use may be associated with improved prognosis in breast cancer patients. Nevertheless, larger size studies are needed to further explore the relationship between beta-blocker drug use and breast cancer prognosis.
\end{abstract}

Keywords: Beta-adrenoceptor blockers - breast cancer - prognosis - systematic review

Asian Pac J Cancer Prev, 16 (18), 8031-8039

\section{Introduction}

Breast cancer is the most common cancer in women in whole world (WHO, 2014). Although treatment of breast cancer has been in progress, global breast cancer mortality is still increasing annually (WHO, 2014). Therefore, new therapeutic options are needed. Recent studies had showed that $\beta$-Adrenergic signaling had an influence up on biological processes involved in the development of the carcinoma (Sood et al., 2006; Thaker et al., 2006; Chakroborty et al., 2009; Anil et al., 2010; Shahzad et al., 2010; Akbar and Alsharidah, 2014) by influencing tumor cells and the microenvironment of tumor cells (Entschladen et al., 2004; Antoni et al., 2006; ArmaizPena et al., 2008; Prema et al., 2008; Susan et al., 2010), including but not limited to the cancers of pancreas (Drell et al., 2003), breast (Barron et al., 2012 ; Diaz et al., 2012), ovary (Masu et al., 2001; Sood et al., 2006), colon (Masur et al., 2001), prostate (Grytli et al., 2013; Grytli et al., 2014). These effects can be removed by BBS (Masur et al., 2001; Drell et al., 2003; Zhang et al., 2010).

Beta-adrenergic system is comprised of catecholamines (Tang et al., 2013), and beta-adrenergic receptor is composed of three subtypes, $\beta 1, \beta 2$ and $\beta 3$ (Yamazaki et al., 2014). In preclinical studies, researchers had observed the expression of beta-adrenergic receptors in a few breast cancer cell lines and human breast cancer tissue samples (Madden et al., 2011; Park et al., 2011; Powe et al., 2011). Beta-adrenergic receptor signaling had been demonstrated to accelerate angiogenesis through increasing the expression of vascular endothelial growth factor (VEGF) (Lutgendorf et al., 2003; Thaker et al., 2006; Yang et al., 2006; Zhang et al., 2010) and interleukin-6 (IL-6) (Thaker et al., 2006; Sloan et al., 2010), promote breast cancer growth (Cakir et al., 2002; Thaker et al., 2006; Shi et al., 2011), recurrence and metastasis (Benish et al., 2008; Avraham et al., 2010; Sloan et al., 2010) and reduce apoptosis of cancer cells (Sastry et al., 2007; Zhang et al., 2009; Sood et al., 2010; Set al., 2011). These effects can be suppressed by BBS (Masur et al., 2001; Drell et al., 2003; Lang et al., 2004; Palm et al., 2006; Landen et al., 2007; Zhang et al., 2010). However, some researchers demonstrated that $\beta 2$-adrenergic receptor stimulation could significantly suppress the malignant transformation of cancers, cancer cell growth and proliferation by cutting of the Raf-1/Mek-1/Erk1/2 pathway (Carie and Sebti, 2007; Perez et al., 2012). In clinical studies, part of authors observed breast cancers' prognosis could be drastically 
improved by receiving BBS (Powe et al., 2010; Barron et al., 2011; Ganz et al., 2011; Melhem et al., 2011; Shah et al., 2011; Botteri et al., 2013; Holmes et al., 2013). Nevertheless some investigators suggested there was no significant relationship between beta-blocker users and non users in the risk of breast cancers' prognosis (Sendur et al., 2012; Holmes et al., 2013). Above all, the result is inconsistent. To make certain whether beta blockers affect the prognosis of breast cancer patients, a systematic review of their relationship is performed.

\section{Materials and Methods}

\section{Criteria for inclusion in this review}

The following inclusion criteria should be met in these candidate studies: (1) the study design was interventional; (2) the breast cancer patients should be confirmed the pathological type; (3) the usage of Beta-adrenoceptor blockers were evaluated; (4) clinical studies used a control group excluding animal and cell line studies; (5) clinical outcomes should be provided, such as survival, diseasefree survival (DFS), tumor recurrence and metastasis. Divergences of the reviewers' inclusions were settled by discussion. Additional studies were identified by a hand search of the references cited in the original studies.

\section{Search methods for identification of studies}

Related literatures are searched from the Pub-Med, EMBASE and Web of nature databases through using terms for breast carcinoma/cancer/tumor and beta-adrenoceptor blockers/beta blockers/beta-adrenoceptor antagonists. The overall search was based on humans and clinic, without restriction on language or region. Furthermore, to extend the search, we also used the "related articles" section. Data retrieval included all eligible studies and their references were thoroughly investigated. These literatures which printed before October 1st, 2014 were in the scope of our screening.

\section{Data extraction}

As listed below, these data was extracted for each study: first author's name, publication year, patients' original country, number of patients investigated including comparison group and experimental group, exposure ascertainment, category of drug use (never/ever use), outcome confirmation, overall risk measurement, adjustments of confounders. The potential biases of all included articles were evaluated. Information was extracted from all eligible studies by two of the authors (Xuesong Chen, Ting Wang). Discrepancies between the authors were settled by consultation to reach a consensus.

\section{Results}

\section{Eligible studies}

There were 2119 potentially relevant studies through electronic search. After checking the title and abstract and screening full text articles when needed, a total of 10 articles were eligible for inclusion criteria as showed in the Figure 1. The main characteristics of these eligible articles are outlined in Table 1. All of the articles were retrospective cohort studies built on the hospital. Five of the studies were located in Europe (Powe et al., 2010; Barron et al., 2011; Shah et al., 2011; Botteri et al., 2013; Cardwell et al., 2013), four in North America (Ganz et al., 2011; Melhem et al., 2011; Holmes et al., 2013; 2013), and one in Asia (Turkey) (Sendur et al., 2012). A total of 19,999 patients were included in the eligible studies, with study sizes ranging from 466 to 9817 . It was variable in terms of beta-adrenoceptor blockers type and the average length of follow up. There were selective and no-selective selective and no-selective beta-adrenoceptor blockers. The average length of follow up ranged from 1.3 years to 10.5 years (Table 1$)$.

\section{Data analysis}

Six studies evaluated the effect of non-selective beta blockers use among breast cancer patients (Ganz et al., 2011; Melhem et al., 2011; Botteri et al., 2013; Cardwell et al., 2013; Holmes et al., 2013; 2013). Four studies assessed the effect of selective and non-selective beta blockers uses (Powe et al., 2010; Barron et al., 2011; Shah et al., 2011; Sendur et al., 2012). One retrospective cohort study provided information on the median daily dose of beta-adrenoceptor blockers $(80 \mathrm{mg}$ ) usage (Barron et al., 2011). Nine studies evaluated the association of Betaadrenoceptor blockers usage versus non-use. Two studies' authors assigned participants into one combined treatment group (BBS and aspirin; BBS and ACEI), a single treatment group (BBS) or a control group (no BBS) (Ganz et al., 2011; Holmes et al., 2013). And Holmes MD et al. (2013) analyzed the association between breast cancer mortality and the use of beta blockers, the combined use of ACEIs and/or aspirin. Ganz et al. (2011) examined the association between BBS Users and BBS combined with angiotensin converting enzyme inhibitors (ACEI) users and breast cancer recurrence, breast cancer specific mortality, and overall mortality. Five studies assessed OS (Ganz et al., 2011; Shah et al., 2011; Melhem et al., 2011; Sendur et al., 2012; Holmes S et al., 2013). Six studies (Powe et al., 2010; Barron et al., 2011; Ganz et al., 2011; Botteri et al., 2013; Holmes et al., 2013; Cardwell et al., 2013) assessed cancer-specific mortality. And two of them assessed overall mortality (Ganz et al., 2011; Botteri et al., 2013). One study assessed RFS (Melhem et al., 2011). Three studies additionally examined tumor

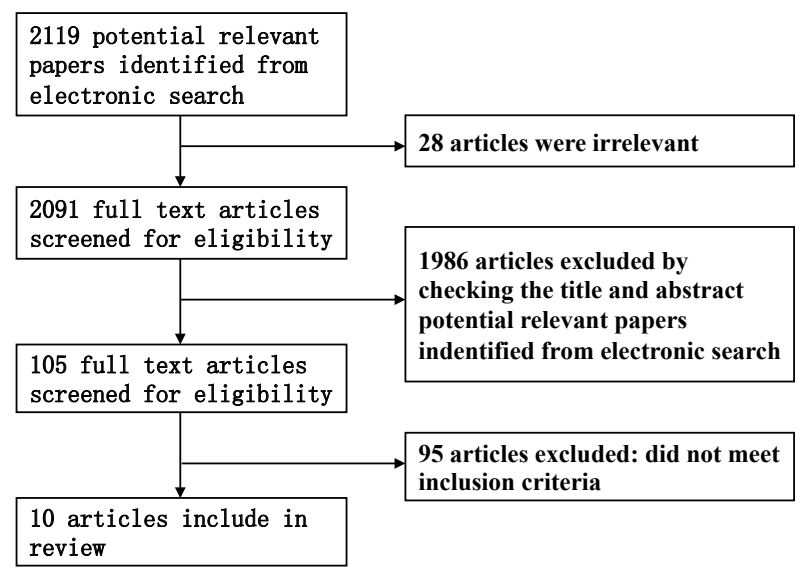

Figure 1. The Flow Chart of Study Selection and Exclusion 
recurrence as an outcome (Powe et al., 2010; Ganz et al., 2011; Botteri et al., 2013). One study assessed disease free interval (Powe et al., 2010). Two studies discussed distant metastasis (DM) (Powe et al., 2010; Botteri et al., 2013). One study assessed DFS ( Sendur et al., 2012). One study observed pathologic complete response (PCR) rates (Melhem et al., 2011).

\section{Risk of adjustments assessment}

Adjustments of these eligible studies were not all the same, which were showed in Table1. Eight of these studies adjusted for age (Powe et al., 2010; Barron et al.,
DOI:http://dx.doi.org/10.7314/APJCP.2015.16.18.8031 $\beta$-adrenergic Receptors : New Target Against Breast Cancer 2011; Ganz et al., 2011; Melhem et al., 2011; Sendur et al., 2012; Botteri et al., 2013; Holmes et al., 2013; Holmes et al., 2013). Seven of the eight studies, moreover, adjusted for stage of cancer simultaneously (Powe et al., 2010; Barron et al., 2011; Ganz et al., 2011; Melhem et al., 2011; Botteri et al., 2013; Holmes et al., 2013; Holmes $\mathrm{S}$ et al., 2013). There were three articles all adjusted for diabetes diagnosis, body mass index (BMI) and hormone receptor status (Ganz et al., 2011; Melhem et al., 2011; Cardwell et al., 2013). Four studies are adjusted for adjuvant treatment (Ganz et al., 2011; Botteri et al., 2013; Cardwell et al., 2013; Holmes et al., 2013). Two studies

Table 1. Characteristics of the Studies

\begin{tabular}{|c|c|c|c|c|c|c|c|c|c|c|c|c|c|c|}
\hline \multirow{2}{*}{$\begin{array}{l}\text { Author } \\
\text { (year) }\end{array}$} & \multirow{2}{*}{$\begin{array}{l}\text { Institution and } \\
\text { country }\end{array}$} & \multirow{2}{*}{$\begin{array}{l}\text { Study } \\
\text { period }\end{array}$} & \multirow{2}{*}{ Study design } & \multirow{2}{*}{$\begin{array}{c}\text { Follow-up } \\
\text { length }\end{array}$} & \multirow{2}{*}{$\begin{array}{l}\text { Statistical } \\
\text { approach }\end{array}$} & \multicolumn{9}{|c|}{ Adjustments } \\
\hline & & & & & & A & B & $\mathrm{C}$ & $\mathrm{D}$ & $\mathrm{E}$ & $\mathrm{F}$ & $\mathrm{G}$ & $\mathrm{H}$ & I \\
\hline $\begin{array}{l}\text { Barron et } \\
\text { al, } 2011\end{array}$ & $\begin{array}{l}\text { National Cancer } \\
\text { registry Ireland } \\
\text { (Ireland) }\end{array}$ & $\begin{array}{l}2001- \\
2006\end{array}$ & $\begin{array}{l}\text { Population- } \\
\text { based } \\
\text { retrospective } \\
\text { cohort }\end{array}$ & 5years & $\begin{array}{c}\text { Conditional } \\
\text { logistic } \\
\text { Regression, } \\
\text { Kaplan-Meier } \\
\text { plots and } \\
\text { Multivariable } \\
\text { Cox proportional } \\
\text { hazards models }\end{array}$ & $*$ & $*$ & & & & & & & * \\
\hline $\begin{array}{l}\text { Ganz et } \\
\text { al, } 2011\end{array}$ & $\begin{array}{c}\text { Kaiser } \\
\text { Permanente } \\
\text { Northern } \\
\text { California Cancer } \\
\text { Registry and } \\
\text { Utah Cancer } \\
\text { Registry (USA) } \\
\end{array}$ & $\begin{array}{l}1997- \\
2010\end{array}$ & $\begin{array}{l}\text { Population- } \\
\text { based } \\
\text { retrospective } \\
\text { cohort }\end{array}$ & 8.2 years & $\begin{array}{c}\text { Pearson } \\
\text { chi-square , } \\
\text { Kruskal-Wallis } \\
(\mathrm{K}-\mathrm{W}) \text { tests and } \\
\text { Cox proportional } \\
\text { hazards model }\end{array}$ & $*$ & $*$ & $*$ & $*$ & $*$ & $*$ & $*$ & & $*$ \\
\hline $\begin{array}{l}\text { Melhem } \\
\text { et al., } \\
2011\end{array}$ & $\begin{array}{c}\text { University } \\
\text { of Texas MD } \\
\text { Anderson Cancer } \\
\text { Center (USA) }\end{array}$ & $\begin{array}{l}1995- \\
2007\end{array}$ & $\begin{array}{l}\text { Hospital-based } \\
\text { retrospective } \\
\text { cohort }\end{array}$ & 5.3 years & $\begin{array}{c}\text { x2 test and } \\
\text { Fisher's exact } \\
\text { test }\end{array}$ & $*$ & $*$ & & $*$ & $*$ & $*$ & $*$ & & $*$ \\
\hline $\begin{array}{l}\text { Botteri et } \\
\text { al., } 2013\end{array}$ & $\begin{array}{l}\text { the European } \\
\text { Institute of } \\
\text { Oncology in } \\
\text { Milan (Italy) }\end{array}$ & $\begin{array}{l}1997- \\
2008\end{array}$ & $\begin{array}{l}\text { Population- } \\
\text { based } \\
\text { retrospective } \\
\text { cohort }\end{array}$ & 3.0 years & $\begin{array}{l}\text { Chi square test, } \\
\text { Fisher exact test, } \\
\text { Gray test and } \\
\text { Multivariable } \\
\text { Cox proportional } \\
\text { hazards models }\end{array}$ & $*$ & $*$ & $*$ & & & & & & * \\
\hline $\begin{array}{l}\text { Powe et } \\
\text { al., } 2010\end{array}$ & $\begin{array}{l}\text { Nottingham City } \\
\text { Hospital (Britain) }\end{array}$ & $\begin{array}{l}1987- \\
1994\end{array}$ & $\begin{array}{l}\text { Hospital-based } \\
\text { retrospective } \\
\text { cohort }\end{array}$ & 1.3 years & $\begin{array}{l}\text { Kaplan-Meier } \\
\text { plots test, Chi } \\
\text { square, Fishers } \\
\text { exact test and } \\
\text { Multivariate } \\
\text { Cox regression } \\
\text { analysis }\end{array}$ & $*$ & & & & & & & & * \\
\hline $\begin{array}{l}\text { Sendur et } \\
\text { al., } 2012\end{array}$ & $\begin{array}{c}\text { Ankara Numune } \\
\text { Education } \\
\text { and Research } \\
\text { Hospital (Turkey) }\end{array}$ & $\begin{array}{l}1998- \\
2010\end{array}$ & $\begin{array}{l}\text { Hospital-based } \\
\text { retrospective } \\
\text { cohort }\end{array}$ & ns & ns & & & & & & & & & \\
\hline $\begin{array}{l}\text { Holmes } \\
\text { MD et } \\
\text { al., } 2013\end{array}$ & $\begin{array}{l}\text { United States } \\
\text { nurses (USA) }\end{array}$ & $\begin{array}{l}1985- \\
2005\end{array}$ & $\begin{array}{l}\text { Population- } \\
\text { based } \\
\text { retrospective } \\
\text { cohort }\end{array}$ & 10.5 years & $\begin{array}{l}\text { Cox propor- } \\
\text { tional hazards } \\
\text { regression and } \\
\text { multivariable } \\
\text { models }\end{array}$ & $*$ & $*$ & $*$ & & $*$ & $*$ & & $*$ & $*$ \\
\hline $\begin{array}{l}\text { Holmes } \\
\text { S et al., } \\
2013\end{array}$ & $\begin{array}{c}\text { Manitoba } \\
\text { Cancer Registry } \\
\text { (Canada) }\end{array}$ & $\begin{array}{l}2004- \\
2008\end{array}$ & $\begin{array}{l}\text { Population- } \\
\text { based } \\
\text { retrospective } \\
\text { cohort }\end{array}$ & 6 years & $\begin{array}{l}\text { Cox regression } \\
\text { analysis and } \\
\text { Kaplan-Meier } \\
\text { survival curves }\end{array}$ & $*$ & $*$ & & & & & & & $*$ \\
\hline
\end{tabular}




\begin{tabular}{|c|c|c|c|c|c|c|c|c|c|c|c|c|}
\hline $\begin{array}{l}\text { Shah et } \\
\text { al., } 2011\end{array}$ & $\begin{array}{c}\text { DIN-LINK } \\
\text { database (Britain) }\end{array}$ & $\begin{array}{l}1997- \\
2007\end{array}$ & $\begin{array}{l}\text { Population- } \\
\text { based } \\
\text { retrospective } \\
\text { cohort }\end{array}$ & 10 years & $\begin{array}{l}\text { Cox proportional } \\
\text { hazard model }\end{array}$ & $*$ & & & & & & \\
\hline $\begin{array}{c}\text { Cardwell } \\
\text { et al., } \\
2013\end{array}$ & $\begin{array}{c}\text { National Cancer } \\
\text { Data Repository, } \\
\text { Clinical Practice } \\
\text { Research } \\
\text { Datalink } \\
\text { and Office } \\
\text { of National } \\
\text { Statistics death } \\
\text { registration data } \\
\text { (Britain) }\end{array}$ & $\begin{array}{l}1998- \\
2007\end{array}$ & $\begin{array}{l}\text { Population- } \\
\text { based } \\
\text { retrospective } \\
\text { cohort }\end{array}$ & 6 years & $\begin{array}{l}\text { Conditional } \\
\text { logistic } \\
\text { regression Cox } \\
\text { proportional } \\
\text { hazards model }\end{array}$ & $*$ & $*$ & $*$ & $*$ & $*$ & $*$ & * \\
\hline
\end{tabular}

*adjusted confounders A age, B stage, C adjuvant treatment, D hormone receptor status, E diabetes, F body mass index, G hypertention, H menopausal statue, I others. ns:not stated

adjusted for hypertension (Ganz et al., 2011; Melhem et al., 2011). Variable adjustments may be having an effect on the above-mentioned studies. However, they were not necessary for a review.

\section{Reported results}

\section{Survival}

It's presented in Table 2 of the risk estimates for survival. Barron et al. (2011) found women taking propranolol at the time of diagnosis $(n=70)$ had an $81 \%$ lower risk of breast cancer-specific mortality ( $\mathrm{HR}=0.19$; 95\% CI=0.06-0.60). However, they observed no association with breast cancer-specific mortality between atenolol and matched nonuser, 26.8\% v26.0\% ( $\mathrm{HR}=1.08$; $95 \% \mathrm{CI}=0.84-1.40)$. After excluding patients with stage IV breast tumors, Propranolol users continued to enjoy a significantly lower risk of breast cancer-specific mortality, compared with matched nonusers $(\mathrm{HR}=0.20 ; 95 \% \mathrm{CI}$ : 0.04 to 0.94$)$. But atenolol users did not $(\mathrm{HR}=1.16 ; 95 \%$ $\mathrm{CI}=0.84$ to 1.61 ). Powe et al. (2010) observed that the risk of breast cancer-specific mortality and metastasis reduced $71 \%(\mathrm{HR}=0.291 ; 95 \% \mathrm{CI}=0.119-0.715 ; p=0.007)$ and $57 \%(\mathrm{HR}=0.430 ; 95 \% \mathrm{CI}=0.200-0.926 ; p=0.031)$, respectively, after 10 years. Botteri et al. (2013) discussed the association BBS in triple-negative breast cancer (TNBC) patients. The population of breast cancer related death was $6(8.1 \%)$ and $141(19.4 \%)$, individually in betablocker users and non-users, with a corresponding adjusted $\mathrm{HR}=0.42$ (95\% CI=0.18-0.97; $p=0.042)$. At univariate analysis, Botteri et al. (2013) observed a suggestion of increased risk of death from causes other than BC in betablocker users compared to non-users, but there was no significant difference. Cardwell et al. (2013) investigated little evidence of an association between beta-blocker use and breast cancer-specific mortality (odds ratio $=0.97$; 95\% CI=0.83, 1.13). Ganz et al. (2011) reported that betablocker users. ACEI users and beta-blocker combined with ACEI users had no statistically significant associated with cause-specific mortality. There was no reduction in overall mortality $(\mathrm{HR}=1.04 ; 95 \% \mathrm{CI}=0.72-1.51)$ in eta-blocker users and $(\mathrm{HR}=1.94 ; 95 \% \mathrm{CI}=1.22,3.10 ; p=0.01)$ in
ACEI users. However, beta blocker combined with ACEI had a higher risk of overall mortality. Powe et al. (2010) showed that beta-blocker treated patients' breast cancer had longer disease free interval $(\mathrm{LR}=6.658 . P=0.011)$ and longer breast cancer specific survival ( $\mathrm{LR}=6.479$; $p=0.011$ ) compared to non treated breast cancer patients in Kaplan-Meier modelling with log rank testing. Modeled simultaneously or individually, the association between the risk of breast cancer death and beta-blocker users was non-significant. Melhem et al. (2011) reported that $\beta$-Blocker use had no significant association with OS among women with ER-positive breast cancer, TNBC, and all breast cancer. Kaplan-Meier estimates of OS showed that the risk of beta-blocker users was significantly improved $(p=0.03)$ in patients with TNBC on beta blockers when compared to TNBC not taking beta blockers. Betablocker users with ER-positive breast cancer had no significant effects on OS $(P=0.65)$. Sendur et al. (2012) observed no statistical significance in 5-year OS rates between metoprolol users and nonusers (metoprolol $96.8 \%$, nonusers $92.9 \% ; p=0.28$ ). Holmes et al. (2013) BBS had no effect on survival for breast cancers (HR= $1.10 ; 95 \% \mathrm{CI}=0.92-1.32 ; p=.305)$. In a population-based retrospective cohort study, Shah et al. (2011) didn't found significant difference in $\mathrm{OS}(\mathrm{HR}=1.09 ; 95 \% \mathrm{CI}=0.80$ $1.49)$ between patients with $\beta$-blocker $(n=434)$ and patients with other antihypertensive medications $(n=554)$. Holmes et al. (2013) observed no significant association with beta blockers and survival.

\section{Disease Progression}

Results for BBS use and disease progression are outlined in Table 2. Botteri et al. (2013) reported that the 5-year cumulative incidence of breast cancer related events (local recurrence, regional recurrence, distant metastases, death from breast cancer) for beta-blocker users and for non-users was $13.6 \%$ and $27.9 \%$ (Gray test $P=0.015$ ), individually. The risk of breast cancer related events was statistically significant for multivariable analysis $(\mathrm{HR}=0.52 ; 95 \% \mathrm{CI}=0.28-0.97 ; p=0.041)$ between beta-blocker users and non-users. The incidence of distant metastases was statistically significant decline 
Table 2. The Relationship Between Beta-adrenoceptor Blockers and Breast Cancer Events

\begin{tabular}{|c|c|c|c|c|c|c|c|c|}
\hline $\begin{array}{c}\text { Author } \\
\text { (year) }\end{array}$ & $\begin{array}{l}\text { Disease } \\
\text { stage }\end{array}$ & Exposure & $\begin{array}{c}\text { Study } \\
\text { population }\end{array}$ & $\begin{array}{c}\text { No. } \\
\text { of } \\
\text { BBS }\end{array}$ & $\begin{array}{l}\text { No. of } \\
\text { controls }\end{array}$ & $\begin{array}{l}\text { Research } \\
\text { objectives }\end{array}$ & $\begin{array}{l}\text { No. of } \\
\text { cases }\end{array}$ & HR \\
\hline $\begin{array}{l}\text { Barron et } \\
\text { al., } 2012 \\
\end{array}$ & I-IV, X & $\begin{array}{c}\text { BBS: } P, \\
\text { Aa }\end{array}$ & 5333 & $\begin{array}{c}\text { P70 } \\
\text { A525 } \\
\end{array}$ & 4738 & BCSD & $\begin{array}{l}P: 4 \text { no: } \\
20\end{array}$ & $\begin{array}{l}P: H R: 0.19 ; 95 \% \text { CI:0.06-0.60 } \\
\text { A:HR:1.08;95\% CI:0.84-1.40 }\end{array}$ \\
\hline \multirow{3}{*}{$\begin{array}{l}\text { Ganz et } \\
\text { al., } 2011\end{array}$} & \multirow{3}{*}{ I-IIIA } & \multirow{3}{*}{$\begin{array}{c}\text { BBS/ } \\
\text { other AH }\end{array}$} & \multirow{3}{*}{1779} & \multirow{3}{*}{204} & \multirow{3}{*}{1372} & BCSD & $\begin{array}{l}\text { User: } 17 \\
\text { no: } 130\end{array}$ & $\begin{array}{c}\text { HR:0.76;95\% CI:0.44- } \\
1.33 ; P: 0.34 \\
\end{array}$ \\
\hline & & & & & & $\begin{array}{l}\text { All cause } \\
\text { mortality }\end{array}$ & $\begin{array}{l}\text { User: } 30 \\
\text { no: } 221\end{array}$ & $\begin{array}{c}\text { HR: } 1.04 ; 95 \% \text { CI:0.74- } \\
1.51 ; P: 0.83\end{array}$ \\
\hline & & & & & & recurrence & $\begin{array}{l}\text { User: } 34 \\
\text { no: } 216\end{array}$ & $\begin{array}{c}\text { HR:0.86;95\% CI:0.57-1.32; } P \\
: 0.49\end{array}$ \\
\hline \multirow{2}{*}{$\begin{array}{l}\text { Melhem et } \\
\text { al., } 2011\end{array}$} & \multirow{2}{*}{ I-III } & \multirow{2}{*}{$\begin{array}{c}\text { BBS/ } \\
\text { other AH }\end{array}$} & \multirow{2}{*}{1413} & \multirow{2}{*}{102} & \multirow{2}{*}{1311} & OS & $\begin{array}{l}\text { User: } 18 \\
\text { no: } 335\end{array}$ & $\begin{array}{c}\text { HRd:0.64, 95\% CI:0.38-1.07; } P \text { : } \\
0.09 \text { HRe:0.35, 95\% CI:0.12- } \\
1.00 ; P: 0.05\end{array}$ \\
\hline & & & & & & RFS & $\begin{array}{l}\text { User: } 17 \\
\text { no: } 387\end{array}$ & $\begin{array}{c}\text { HRd:0.52, 95\% CI:0.31- } \\
0.88 ; P: 0.015 \text { HRe: } 0.30,95 \% \\
\text { CI:0.10-0.87;P:0.027 }\end{array}$ \\
\hline \multirow{3}{*}{$\begin{array}{l}\text { Botteri et } \\
\text { al., } 2013\end{array}$} & \multirow{3}{*}{ I-IVb } & \multirow{3}{*}{$\begin{array}{c}\text { BBS/ } \\
\text { other AH }\end{array}$} & \multirow{3}{*}{800} & \multirow{3}{*}{74} & \multirow{3}{*}{726} & BCSD & $\begin{array}{l}\text { User: } 6 \\
\text { no: } 141 \\
\end{array}$ & $\begin{array}{c}\text { HR:0.42, 95\% CI:0.18-0.97;P: } \\
0.042 \\
\end{array}$ \\
\hline & & & & & & $\begin{array}{l}\text { Breast } \\
\text { cancer } \\
\text { related } \\
\text { events }\end{array}$ & $\begin{array}{l}\text { User: } 11 \\
\text { no: } 199\end{array}$ & $\begin{array}{c}\text { HR: } 0.52 ; 95 \% \text { CI:0.28- } \\
0.97 ; p: 0.041\end{array}$ \\
\hline & & & & & & $\mathrm{DM}$ & $\begin{array}{l}\text { User: } 3 \\
\text { no: } 110\end{array}$ & $\begin{array}{c}\text { HR:0.32, 95\% CI:0.12-0.90;P: } \\
0.031\end{array}$ \\
\hline \multirow{6}{*}{$\begin{array}{l}\text { Powe et } \\
\text { al., } 2010\end{array}$} & \multirow{6}{*}{ I-II } & \multirow{6}{*}{$\begin{array}{c}\text { BBS/ } \\
\text { other AH }\end{array}$} & \multirow{6}{*}{466} & \multirow{6}{*}{43} & \multirow{6}{*}{374} & BCSD & ns & $\begin{array}{c}\text { HRf:0.291;95\% CI:0.119-0.715, } \\
p: 0.007\end{array}$ \\
\hline & & & & & & $\begin{array}{l}\text { specific } \\
\text { survival }\end{array}$ & ns & LR:6.479, $p: 0.011$ \\
\hline & & & & & & $\begin{array}{c}\text { Disease } \\
\text { free interval } \\
\end{array}$ & ns & LR:6.658, $p: 0.011$ \\
\hline & & & & & & \multirow{2}{*}{ DM } & $\begin{array}{l}\text { User: } 5 \\
\text { NH: } 102 \\
\end{array}$ & $\begin{array}{c}\text { HRf:0.430;95\% CI:0.200-0.926, } \\
\quad p: 0.031 \text { x2g:4.852, } p: 0.028\end{array}$ \\
\hline & & & & & & & $\begin{array}{c}\text { Other } \\
\text { AH: } 15\end{array}$ & $\mathrm{x} 2: 7.264 p: 0.026$ \\
\hline & & & & & & recurrence & ns & \\
\hline \multirow{2}{*}{$\begin{array}{l}\text { Sendur et } \\
\text { al., } 2012\end{array}$} & \multirow{2}{*}{ I-III } & \multirow{2}{*}{$\begin{array}{l}\text { BBS } \\
\text { (meto- } \\
\text { prolol) }\end{array}$} & \multirow{2}{*}{544} & \multirow{2}{*}{88} & \multirow{2}{*}{456} & OS & ns & User:96.8\%, no:92.9\% P:0.28 \\
\hline & & & & & & DFS & ns & User:98.3\%, no:90.4\% P:0.13 \\
\hline $\begin{array}{l}\text { Holmes } \\
\text { MD et al., } \\
2013\end{array}$ & I-III & $\begin{array}{c}\text { BBS/ } \\
\text { other AH }\end{array}$ & 4661 & 770 & 3891 & BCSD & $\begin{array}{l}\text { User: } 45 \\
\text { no: } 247\end{array}$ & HR:0.76, 95\% CI:0.54-1.05 \\
\hline $\begin{array}{l}\text { Holmes S } \\
\text { et al., } 2013\end{array}$ & I-IV & $\begin{array}{c}\text { BBS/ } \\
\text { other AH }\end{array}$ & 4019 & 531 & 2310 & Survival & ns & HR: $1.10,95 \%$ CI: 0.92-1.32 \\
\hline $\begin{array}{l}\text { Shah et al., } \\
2011\end{array}$ & ns & $\begin{array}{c}\text { BBS/ } \\
\text { other AH }\end{array}$ & 984 & 434 & 554 & OS & ns & HR:1.09;95\% CI: $0.80-1.49$ \\
\hline $\begin{array}{l}\text { Cardwell } \\
\text { et al., } 2013\end{array}$ & I-IV & BBS & 9817 & 1378 & 5754 & BCSD & $\begin{array}{l}\text { User: } \\
182 \text { no: } \\
677\end{array}$ & HR: $1.24,95 \%$ CI:0.81, 1.88 \\
\hline
\end{tabular}

ns: not stated AH: antihypertensive therapy DM: distant metastases; NH: Non hypertensive breast cancer patients RFS: relapse-free survival DFS: disease-free survival BCSD: breast cancer specific death a $P$ : propranolol A: atenolol ; b: Patients with metastatic disease at diagnosis were excluded; c: Pre-diagnostic beta-blocker use in 2 years prior to breast cancer diagnosis; d: all breast cancer patients; e: Triple-negative breast cancer patients; f: compared to Non -treated breast cancer patients; g: compared to patients receiving other $\mathrm{AH}$

$(\mathrm{HR}=0.32 ; 95 \% \mathrm{CI}=0.12-0.90 ; p=0.031)$ in favor of BBS. In the study by Powe et al. (2010), the authors reported a significant reduction in metastasis development $\left(\chi^{2}=4.986\right.$; $p=0.026)$. In addition, when analyzing the risk of distant metastases, they observed a significant difference between beta-blocker drugs users and other anti-hypertensive 
medications users, $11.6 \%$ v30.6\% ( $\left.\chi^{2}=4.852 ; p=0.028\right)$. In Kaplan-Meier modeling with log rank testing, hypertensive breast cancer patients therapeutically treated with BBS users owned significantly longer distant metastasis-free interval $(\log \operatorname{rank}(\mathrm{LR})=5.208 ; p=0.022)$ compared to non-treated patients. At a multivariate Cox hazard model, the author demonstrated that the risk of developing DM decreased 57\% ( $\mathrm{HR}=0.430$; $\mathrm{CI}=0.200$ $0.926 ; p=0.031)$ in beta-blocker treated patients compared to non-treated breast cancer patients. Melhem et al. (2011) found that beta-blocker users achieved a markedly improved RFS among all breast cancer patients $(\mathrm{HR}=0.52$; 95\% CI=0.31-0.88; $p=0.015)$ and patients with TNBC (HR=0.30; 95\% CI=0.10-0.87; $p=0.027)$. Beta-Blocker users, however, had no significant association with RFS among women with ER-positive breast cancer. In addition, When compared beta-blocker users to non-users by the Kaplan-Meier estimates of RFS, the RFS was markedly improvement $(p=0.02)$ among patients with TNBC on BBS, but not among ER-positive breast cancer $(P=0.4)$. Sendur et al. (2012) didn't find statistical significance in DFS (metoprolol 98.3\% V nonusers 90.4\%, $p=0.13$ ), when compare metoprolol users to nonusers. In the study of Powe et al. (2010), the authors reported a significant reduction in tumor recurrence between BBS users and nonhypertensive breast cancer patients $\left(\chi^{2}=13.091 ; p=0.001\right)$, and between other anti-hypertensive medications patients and BBS users $\left(\chi^{2}=7.264 ; p=0.026\right)$. Ganz et al. (2011) reported patients with $\beta$-blockers showed a non-significant $14 \%$ reduction $(\mathrm{HR}=0.86 ; 95 \% \mathrm{CI}=0.57-1.32)$ in the risk of breast cancer recurrence (distant, loco-regional, or contralateral) compared to unexposed women (no BBS and ACEI). Melhem et al. (2011) found no significant $48 \%$ and $70 \%$ reduction in risk of breast cancer recurrence in beta users of TNBC and breast cancer, respectively. Botteri et al. (2013) also observed that the local and regional recurrence was no significant change between BBS users and non-users.

\section{Discussion}

Although the relationship between beta-adrenoceptor blockers and breast-carcinoma events has been observed in previous studies, the conclusions were inconsistent and some were even controversial. So a systematic review was needed in order to investigate the further association. To the best of our knowledge, this is the first systematic review to investigate the association between Betaadrenoceptor blockers and breast-carcinoma events. Ten retrospective cohort studies were identified. The majority of the eligible studies were from hospital. Most studies were in small size and had relatively short follow-up periods.

Three studies reported a significant reduction in the risk of breast cancer-specific mortality in beta-blocker users (Powe et al., 2010; Barron et al., 2011; Botteri et al., 2013). Furthermore, one of them observed a no significant improvement of the mortality from causes other than breast cancer in beta-blocker users (Botteri et al., 2013). Three studies demonstrated a suggestion of a reduced risk of breast cancer death associated with beta-blocker drugs, but not reached statistical significance (Ganz et al., 2011; Cardwell et al., 2013; Holmes et al., 2013). In these researchers, Ganz et al. (2011), Cardwell et al. (2013) and Holmes et al. (2013) adjusted most of the adjustments. Thomas I. Barron, Powe et al. (2010) and Botteri et al. (2013) only adjusted others, age, stage, and adjustment treatment. Powe et al. (2010) observed a significant association between breast cancer special survival and beta-adrenoceptor blockers. And only age and other confounding factors were adjusted in this literature.One study found no significant $36 \%$ and $65 \%$ reduction in risk of OS in beta blocker users with TNBC and breast cancer, respectively. However, the OS $(P=0.03)$ of patients with TNBC on beta blockers were significantly improved in the Kaplan-Meier estimates (Melhem et al., 2011). The different statistical methods maybe affect the results. Three studies found no significant association between OS and beta-adrenoceptor blockers, and they also only adjusted age, stage, and other confounding factors (Shah et al., 2011; Sendur et al., 2012; Holmes et al., 2013). The risk of distant metastases was assessed in two studies. And both of them demonstrated a significant reduction in the risk of distant metastases in beta-blocker treated patients compared to non-treated patients (Powe et al., 2010; Botteri et al., 2013). One study demonstrated a significant reduction in the risk of breast cancer recurrence (Powe et al., 2010), and three studies observed no significant reduction (Ganz et al., 2011; Melhem et al., 2011; Botteri et al., 2013). Ganz et al. (2011) observed no significant $14 \%$ reduction. Powe et al. (2010), Melhem et al. (2011) and Botteri et al. (2013) adjusted the multiple confounding factors. Powe et al. (2010) adjusted two confounding factors. Hormone receptor status, age, stage, hypertension, menopausal status, adjuvant treatment, diabetes and BMI were important prognostic indicators. Recently, it's demonstrated that diabetes was an adverse factor for breast cancer (Liao et al., 2011; Melhem et al., 2011; De et al., 2013; Xiao et al., 2014), and so did BMI (Ortiz-Mendoza et al., 2014). A diagnosis of diabetes was associated with a worse OS $(P=0.002)$ but not a worse RFS $(P=0.42)$ (Melhem et al., 2011). Information about performance status (Huber et al., 2002; Van et al., 2011; Werner and Bruchim, 2012), physical activities (Akechi et al., 2011; Ying et al., 2013) and hereditary factors were not adjusted in the majority of studies, which were reported as important factors for cancer prognosis. Adjustment factors of the eligible studies were inconsistent, which maybe have an effect on the risk of breast cancer-specific mortality. There were only five articles referring to selective and non-selective beta blockers (Powe et al., 2010; Barron et al., 2011; Shah et al., 2011; Sendur et al., 2012; Cardwell et al., 2013). The consequences, however, were inconsistent. Only one of these studies reported that the different strategies of beta-adrenoceptor blockers showed a significantly different effect on breast cancer prognosis (Barron et al., 2011).

Potential biases or confounding. First, although we try our best to search all possible relevant data, it was unavoidable to miss data. Second, a part of the eligible studies was observational studies whose observations may have potential bias. Third, patients' information 
was obtained from hospital medical records in the cohort studies, which may appear incomplete information for patients due to differences in hospital classification of drugs and other ways to obtain drugs. Four, there are unavoidable differences in the definitions of methods, measurements, and outcomes in the eligible studies. Despite these potential biases or confounding, this systematic review had several noteworthy advantages. First, this is the first systematic review to investigate the association between BBS use and breast cancer progression and survival. Second, due to a considerable number of subjects and cases from different studies, the statistical power of the analysis was significantly increased. Third, all the eligible studies were searched from aforementioned databases, and when there were discrepancies about candidate articles, the articles were discussed and independently screened for inclusion criteria.

Because of the small number of selected articles and the heterogeneity of articles, a meta-analysis was not undertaken. In this systematic review, it's demonstrated that the risk of breast cancer-specific mortality, the risk of DM and the risk of breast cancer recurrence were decreased for BBS users when compared to non-users, despite the fact that some of the data was without statistical significance. Compared with non-users, the OS of breast cancer patients with beta blockers and different type of beta blockers was inconsistent. And the possible influencing factors could be the following. Firstly, the size of some studies' samples are not enough large; secondly, the discrepancy of the data analysis methods may influence the result; thirdly, other inconsistent confounders of the patients perhaps have some effect on the OS; fourthly, compared with non-users, BBS users were more likely to combine with other diseases which may have a influence on breast cancer prognosis and cancer therapy.

\section{Conclusions}

From this system review, it's suggested that betaadrenoceptor blocking therapy improve breast cancer prognosis. However, it's not fit to reach a definitive conclusion on the effect of beta-adrenoceptor blocking therapy on the breast cancer prognosis. More and more large preclinical studies were needed in order to further investigate the association between beta- adrenoceptor blockers and breast cancer. Meanwhile, more clinical studies on the association between breast cancer progression or survival and the dosage, frequency and duration of use of beta blockers were also needed to be further carried out.

\section{Acknowledgements}

This research is supported by Heilongjiang Provincial Department of Education Project (No. 12541435 to CX); National Natural Science Foundation (81573001 to CX); Harbin Medical University Cancer Hospital haiyan Scientific Research Foundation (No.JJLX2014-02 to CX); Heilongjiang Province Foundation for returness (LC201438 to CX); Heilongjiang Province Administration of Traditional Chinese Medicine (ZQG-045 to CX).

\section{References}

World Health Organization (WHO) (2014): Breast cancer: prevention and control. Retrieved from $<\mathrm{http} / / / \mathrm{www}$.who. $\mathrm{int} /$ cancer/detection/breast cancer/en/>.

Akbar S1, Alsharidah MS (2014). Are beta blockers new potential anticancer agents? Asian Pac J Cancer Prev, 15, 9567-74.

Akechi T1, Okuyama T, Endo C, et al (2011). Patient's perceived need and psychological distress and/or quality of life in ambulatory breast cancer patients in Japan. Psychooncol, 20, 497-505.

Anil K. Sood, Guillermo N. Armaiz-Pena, et al (2010). Adrenergoc modulation of adhesion kinase protects human ovarian cells from anoikis. J Clin Invest, 120, 1515-23.

Antoni MH, Lutgendorf SK, Cole SW, et al (2006). The influence of bio-behavioural factors on tumour biology: Pathways and mechanisms. Nat Rev Cancer, 6, 240-8.

Armaiz-Pena GN, Lutgendorf SK, Cole SW, Sood AK (2009). Neuroendocrine modulation of cancer progression. Brain Behav Immun, 23, 10-5.

Avraham R, Benish M, Inbar S, et al (2010). Synergism between immunostimulation and prevention of surgery-induced immune suppression: an approach to reduce post-operative tumor progression. Brain Behav Immun, 24, 952-8.

Barron TI, Connolly RM, Sharp L, Bennett K, Visvanathan K (2011). Beta blockers and breast cancer mortality: a population based study. J Clin Oncol, 29, 2635-44.

Barron TI, Sharp L, Visvanathan K (2012). Beta-adrenergic blocking drugs in breast cancer: a perspective review. Ther Adv Med Oncol, 4, 113-25.

Benish M, Bartal I, Goldfarb Y, et al (2008). Perioperative use of beta-blockers and COX-2 inhibitors may improve immune competence and reduce the risk of tumor metastasis. Ann Surg Oncol, 15, 2042-52.

BotteriE, Munzone E, Rotmensz N, et al (2013). Therapeutic effect of $\beta$-blockers in triple-negative breast cancerpostmenopausal women. Breast Cancer Res Treat, 140, 567-75.

Cakir Y, Plummer HK 3rd, Tithof PK, Schuller HM (2002). Betaadrenergic and arachidonic acid-mediated growth regulation of human breast cancer cell lines. Int J Oncol, 21, 153-7.

Cardwell CR, Coleman HG, Murray LJ, et al (2013). Betablocker usage and breast cancer survival: a nested casecontrol study within a UK clinical practice research datalink cohort. Int J Epidemiol, 42, 1852-61.

Carie AE, Sebti SM (2007). A chemical biology approach identifies a beta-2 adrenergic receptor agonist that causes human tumor regression by blocking the Raf-1/ Mek-1/ Erk1/2 pathway. Oncogene, 26, 3777-88.

Chakroborty D, Sarkar C, Basu B, Dasgupta PS, Basu S (2009). Catecholamines regulate tumor angiogenesis. Cancer Res, 69, 3727-30.

De Bruijn KM, Arends LR, Hansen BE, et al (2013). Systematic review and meta-analysis of the association between diabetes mellitus and incidence and mortality in breast and colorectal cancer. Br J Surg, 100, 1421-9.

Diaz ES, Karlan BY, Li AJ (2012). Impact of beta blockers on epithelial ovarian cancer survival. Gynecol Oncol, 127, 375-8.

Drell TL 4th, Joseph J, Lang K, et al (2003). Effects of neurotransmitters on the chemokinesis and chemotaxis of MDA-MB-468 human breast carcinoma cells. Breast Cancer Res Treat, 80, 63-70.

Entschladen F, Drell TL 4th, Lang K, Joseph J, Zaenker KS (2004). Tumour-cell migration, invasion, and metastasis: Navigation by neurotransmitters. Lancet Oncol, 5, 254-8.

Fidler IJ (2003). The pathogenesis of cancer metastasis: The 
Ting Wang et al

'seed and soil' hypothesis revisited. Nat Rev Cancer, 3, 453-8.

Ganz PA, Habel LA, Weltzien EK, Caan BJ, Cole SW (2011). Examining the Influence of Beta Blockers and ACE Inhibitors on the Risk for Breast Cancer Recurrence: Results from the LACE Cohort. Breast Cancer Res Treat, 129, 549-56.

Grytli HH, Fagerland MW, Fossa SD, Tasken KA, Haheim LL (2013). Use of beta-blockers is associated with prostate cancer-specific survival in prostate cancer patients on androgen deprivation therapy. Prostate, 73, 250-60.

Grytli HH, Fagerland MW, Fossa SD, Tasken KA (2014). Association between use of $\beta$-blockers and prostate cancerspecific survival: a cohort study of 3561 prostate cancer patients with high-risk or metastatic disease. Eur Uro, $\mathbf{6 5}$, 635-41.

Holmes MD, Hankinson SE, Feskanich D, Chen WY (2013). Beta blockers and angiotensin-converting enzyme inhibitors' purported benefit on breast cancer survival may be explained by aspirin use. Breast Cancer Res Treat, 139, 507-13.

Holmes S, Griffith EJ, Musto G, Minuk GY (2013). Antihypertensive medications and survival in patients with cancer: A population-based retrospective cohort study. Cancer Epidemiol, 37, 881-5.

Huber JC1, Schneeberger C, Tempfer CB (2002). Genetic modelling of the estrogen metabolism as a risk factor of hormone-dependent disorders. Maturitas, 42, 1-12.

Landen CN Jr, Lin YG, Armaiz Pena GN, et al (2007). Neuroendocrine modulation of signal transducer and activator of transcription-3 in ovarian cancer. Cancer Res, 67, 10389-96.

Lang K, Drell TL 4th, Lindecke A, et al (2004). Induction of a metastatogenic tumor cell type by neurotransmitters and its pharmacological inhibition by established drugs. Int $J$ Cancer, 112, 231-8.

Liao S1, Li J, Wei W, Wang L, et al (2011). Association between diabetes mellitus and breast cancer risk: a meta-analysis of the literature. Asian Pac J Cancer Prev, 12, 1061-5.

Lutgendorf SK, Cole S, Costanzo E, et al (2003). Stress-related mediators stimulate vascular endothelial growth factor secretion by two ovarian cancer cell lines. Clin Cancer Res, 9, 4514-21.

Madden, K.S., Szpunar, M.J. and Brown, E.B (2011). $\beta$-Adrenergic receptors $(\beta$-AR) regulate VEGF and IL-6 production by divergent pathways in high $\beta$-AR-expressing breast cancer cell lines. Breast Cancer Res Treat, 130, 747-58.

Masur K, Niggemann B, Zanker KS, Entschladen F (2001). Norepinephrine-induced migration of SW 480 colon carcinoma cells is inhibited by beta-blockers. Cancer Res, 61, 2866-9.

Melamed R, Rosenne E, Shakhar K, et al (2005). Marginating pulmonary-NK activity and resistance to experimental tumor metastasis: suppression by surgery and the prophylactic use of a beta-adrenergic antagonist and a prostaglandin synthesis inhibitor. Brain Behav Immun, 19, 114-26.

Melhem-Bertrandt A, Chavez-Macgregor M, Lei X, et al (2011). Beta-blocker use is associated with improved relapse-free survival in patients with triple-negative breast cancer. JClin Oncol, 29, 2645-52.

Ortiz-Mendoza CM, de-la-Fuente-Vera TA, Perez-Chavez E (2014). Metabolic syndrome in Mexican women survivors of breast cancer: a pilot study at a general hospital. Med Arch, 68, 19-21.

Palm D, Lang K, Niggemann B, et al (2006). The norepinephrinedriven metastasis development of PC-3 human prostate cancer cells in BALB/c nude mice is inhibited by beta- blockers. Int J Cancer, 118, 2744-9.

Park SY, Kang JH, Jeong KJ, et al (2011). Norepinephrine induces VEGF expression and angiogenesis by a hypoxiainducible factor- $1 \alpha$ protein-dependent mechanism. Int $J$ Cancer, 128, 2306-16.

Perez Piñero C, Bruzzone A, Sarappa MG, Castillo LF, Lüthy IA (2012). Involvement of $\alpha 2$ - and $\beta 2$-adrenoceptors on breast cancer cell proliferation and tumour growth regulation. $\mathrm{Br}$ $J$ Pharmacol, 166, 721-36.

Powe DG, Voss MJ, Zänker KS, et al (2010). Beta-blocker drug therapy reduces secondary cancer formation in breast cancer and improves cancer specific survival. Oncotarget, 1, 628-38.

Powe DG, Voss MJ, Habashy HO, et al (2011). Alpha- and beta-adrenergic receptor (AR) protein expression is associated with poor clinical outcome in breast cancer: an immunohistochemical study. Breast Cancer Res Treat, 130, 457-63.

Premal H. Thaker, Anil K. Sood (2008). The Neuroendocrine Impact of Chronic Stress on Cancer. Semin Cancer Biol, 18, 164-70.

Ronco AL1, De Stefani E, Deneo-Pellegrini H, Quarneti A (2012). Diabetes, overweight and risk of postmenopausal breast cancer: a case-control study in Uruguay. Asian Pac J Cancer Prev, 13, 139-46.

Sastry KS, Karpova Y, Prokopovich S, et al (2007). Epinephrine protects cancer cells from apoptosis via activation of cAMPdependent protein kinase and BAD phosphorylation. J Biol Chem, 282, 14094-100.

Shah SM, Carey IM, Owen CG, et al (2011). Dose $\beta$-adrenoceptor blocker therapy improve cancer survival? findings from a population-based retrospective cohort study. $\mathrm{Br} \mathrm{J}$ Clin Pharmacol, 72, 157-61.

Shahzad MM, Arevalo JM, Armaiz-Pena GN, et al (2010). Stress effects on FosB- and interleukin-8 (IL8)-driven ovarian cancer growth and metastasis. J Biol Chem, 285, 35462-70.

Shan T, Ma Q, Zhang D, et al (2011). ß2-Adrenoceptor blocker synergizes with gemcitabine to inhibit the proliferation of pancreatic cancer cells via apoptosis induction. Eur $J$ Pharmacol, 665, 1-7.

Shi M, Liu D, Duan H, et al (2011). The $\beta 2$-adrenergic receptor and Her2 comprise a positive feedback loop in human breast cancer cells. Breast Cancer Res Treat, 125, 351-62.

Sloan EK, Priceman SJ, Cox BF, et al (2010). The sympathetic nervous system induces a metastatic switch in primary breast cancer. Cancer Res, 70, 7042-52.

Sood AK, Bhatty R, Kamat AA, et al (2006). Stress hormonemediated invasion of ovarian cancer cells. Clin Cancer Res, 12, 369-75.

Sood AK, Armaiz-Pena GN, Halder J, et al (2010). Adrenergic modulation of focal adhesion kinase protects human ovarian cancer cells from anoikis. J Clin Invest, 120, 1515-23.

Susan K. Lutgendorf, Anil K. Sood, Michael H. Antoni (2010). Host factors and cancer progression: Biobehavioral signaling pathways and interventions. J Clin Oncol, 28, 4094-9.

Tang J1, Li Z, Lu L, Cho CH (2013). Seminars in Cancer Biology $\beta$-Adrenergic system, a backstage manipulator regulating tumour progression and drug target in cancer therapy. Semin Cancer Biol, 23, 533-42.

Thaker PH, Han LY, Kamat AA, et al (2006). Chronic stress promotes tumor growth and angiogenesis in a mouse model of ovarian carcinoma. Nat Med, 12, 939-44.

van Zitteren M1, van der Net JB, Kundu S, et al (2011). Genome-based prediction of breast cancer risk in the general population: a modeling study based on meta-analyses of genetic associations. Cancer Epidemiol Biomarkers Prev, 20, 9-22.

Werner H1, Bruchim I (2012). IGF-1 and BRCA1 signalling 
pathways in familial cancer. Lancet Oncol, 13, 537-44.

Xiao Y, Zhang S, Hou G, et al (2014). Clinical pathological characteristics and prognostic analysis of diabetic women with luminal subtype breast cancer. Tumour Biol, 35, 203545.

Yamazaki S, Miyoshi N, Kawabata K, et al (2014). Quercetin3-O-glucuronide inhibits noradrenaline-promoted invasion of MDA-MB-231 human breast cancer cells by blocking $\beta 2$-adrenergic signaling. Arch Biochem Biophys, 557, 18-27.

Yang EV, Bane CM, MacCallum RC, et al (2002). Stress-related modulation of matrix metalloproteinase expression. $J$ Neuroimmu- nol, 133, 144-50.

Yang EV, Sood AK, Chen M, et al (2006). Norepinephrine up-regulates the expression of vascular endothelial growth factor, matrix metal- loproteinase (MMP)-2, and MMP-9 in nasopharyngeal carcinoma tumor cells. Cancer Res, $\mathbf{6 6}$, 10357-64.

Ying Gao, Yu-Bei Huang, Xue-Ou Liu, et al (2013). Tea consumption, alcohol drinking and physical activity associations with breast cancer risk among chinese females: a systematic review and meta-analysis. Asian Pac J Cancer Prev, 14, 7543-50.

Zhang D, Ma Q, Shen S, Hu H (2009). Inhibition of pancreatic cancer cell proliferation by propranolol occurs through apoptosis induction: the study of beta-adrenoeptor antagonist's anticancer effect in pancreatic cancer cell. Pancreas, 38, 94-100.

Zhang D, Ma QY, Hu HT, Zhang M (2010). Beta2-adrenergic antagonists suppress pancreatic cancer cell invasion by inhibiting CREB, NFkB and AP-1. Cancer Biol Ther, 10, 19-29. 